\title{
Can HbA1c act as a surrogate marker for cardiovascular risk?
}

\author{
Sasisekhar T.V.D ${ }^{1}$, Shabana $\mathrm{S}^{2}$ \\ ${ }^{I}$ Assoc Prof, Department of medicine, Dr.PSIMS \& RF Chinna Avutapalli, Vijayawada, Andhra Pradesh \\ ${ }^{2}$ Assoc Professor of Biochemistry, Dr. PSIMS \& RF, Chinna Avutapalli, Vijayawada, Andhra Pradesh
}

\begin{abstract}
AIM:- Glycosylated hemoglobin is a used marker for long-term glycemic control.This investigation is an attempt to evaluate the diagnostic value of $\mathrm{Hb} A(1 \mathrm{lc})$ in predicting cardiovascular risk

MATERIALS AND METHODS: The study was conducted in Dr PSIMS\&RF,Vijayawada. 278 subjects during May 2012 to October 2012, were included in the study

Details regarding to age, gender, duration of diabetes, were taken. Venous blood samples were collected and analyzed for glycosylated hemoglobin (HbAlc), fasting plasma glucose (FPG) , Total Cholesterol, Triglycerides, $H D L-C$ and $L D L-C$ and $A I P$

RESULTS: The total cholesterol and LDL cholesterol were significantly higher in females as compared to male diabetics. TG levels and AIP ratio were raised in the subjects $<55$ years compared to those $>55$ years. In cases with duration of diabetes > 6 years $\mathrm{HbAlc}$, total cholesterol, $T G, L D L$ cholesterol and AIP ratio were elevated but only total cholesterol and LDL cholesterol were statistically significant. All the lipid parameters except $H D L$ cholesterol were higher in subjects with HbAlc $>7 \%$ as compared to the group with HbAlc $<7 \%$.

CONCLUSION: The findings indicate that HbAlc can provide auxiliary information about the extent of circulating lipids and AIP, and can thus be used as a predictor of cardiovascular risk in diabetics
\end{abstract}

Keywords: Diabetes, Dyslipidemia, HbAlc, AIP

\section{Introduction}

The worldwide prevalence of diabetes mellitus has increased dramatically in the past 20 years. India now stands only second to china in the largest number of diabetic patients in the world. In 2011 there were 366 million people with diabetes, and these are expected to rise to 552 million by $2030^{[1]}$. The burden of diabetes is to a large extent the consequence of macrovascular (coronary artery disease, peripheral vascular disease, and atherosclerosis) and microvascular (retinopathy, neuropathy and nephropathy) complications of the disease ${ }^{[2]}$.

The percentage of glycosylated hemoglobin $(\mathrm{HbAlc})$ reflects the glycemic control of a patient during the 8-10 week period before the blood sample was obtained. The Diabetes complications and control trial (DCCT) established $\mathrm{HbA} 1 \mathrm{c}$ as the gold standard of glycemic control. Lowering $\mathrm{HbA1C}$ has been shown to reduce microvascular complications of diabetes, and if implemented soon after the diagnosis of diabetes, is associated with long-term reduction in macrovascular disease ${ }^{[3,10]}$.

Dyslipidemia is one of the major established risk factors for cardiovascular disease in diabetes mellitus $^{[4]}$. The characteristic features of diabetic dyslipidemia are a high plasma triglyceride concentration, low HDL cholesterol concentration and increased concentration of small dense LDL-cholesterol particles. The Atherogenic index of plasma has recently been proposed as a marker of plasma atherogenicity and is positively correlated with cardiovascular disease risk ${ }^{[5]}$.

Apart from classical risk factors like dyslipidemia, elevated $\mathrm{HbA} 1 \mathrm{c}$ is now regarded as an independent risk factor for CVD in subjects with or without diabetes ${ }^{[6]}$. HbA1c is directly related to the severity of coronary artery disease (CAD) in diabetic patients. Estimated risk of CAD has shown to be increased by $18 \%$ for each $1 \%$ increase in absolute HbA1c value in diabetic population ${ }^{[7]}$.

Moreover, attempts to reduce cardiovascular risks resulted in the improvement of $\mathrm{HbA1c}$ even in the absence of any specific intervention targeted at improving glycemic contro ${ }^{[8]}$. A soluble form of receptor for advanced glycation end products (sRAGE) in type 2 diabetic patients with CAD was found to be elevated with significant association between sRAGE and HbA1c as well as serum lipids ${ }^{[9]}$.

In view of the predisposition for the development of atherosclerotic vascular disease in the diabetics, attention has been focused on glycemic control, lipid and lipoprotein levels in diabetics. This investigation is an attempt to evaluate the diagnostic value of $\mathrm{HbA} 1 \mathrm{c}$ in predicting cardiovascular risk

\section{Material And Methods:}

The study was conducted in Dr Pinnamaneni Siddhartha Institute of Medical Sciences and Research Foundation, a rural tertiary health care centre after obtaining approval from the Institutions ethical committee. Verbal consent was obtained from each respondent and the ethical committee approved the procedure since the study was a survey and with no any harm to the respondents. 
All type 2 diabetic subjects attending department of medicine during May 2012 to October 2012 were included in to the study. A Systematic Random Sampling (every third subject) was taken as the mode of selection. A total of 278 subjects were recruited in the study.

Subjects in the age group of 35-70 years of both sexes and those who gave a history of diabetes and were under treatment with either oral antidiabetic drugs or insulin were included in the study. The subjects who gave a history of any cardiovascular, renal or thyroid disorders were excluded from the study.

Details pertaining to the age, gender, duration of diabetes, lipid profile and HbA1c were taken. Venous blood samples from all the subjects were collected after at least $8 \mathrm{~h}$ fasting and analyzed for glycosylated hemoglobin (HbA1c), fasting plasma glucose (FPG), serum total cholesterol(TC), triglycerides (TG) and highdensity lipoprotein cholesterol (HDL-C) on Randox (Daytona) autoanalyzer. The level of low- density lipoprotein cholesterol (LDL-C) was determined using the formula: LDL= Total Cholesterol $-(\mathrm{TG} / 5+\mathrm{HDL})$.

Operational Definitions: HbA1c of $<7 \%$ was considered good glycemic control, while a $\mathrm{HbA} 1 \mathrm{c}>7 \%$ was considered as poor glycemic control. The selection of these cutoff values of HbA1c was based on earlier studies $^{[10]}$.

For serum lipid reference level, National Cholesterol Education Programme (NCEP) Adult Treatment Panel III (ATP III) guideline was referred. According to NCEP-ATPIII guideline, hypercholesterolemia is defined as TC > $200 \mathrm{mg} / \mathrm{dl}$, high LDL-C when value $>100 \mathrm{mg} / \mathrm{dl}$, hy-pertriglyceridemia as TAG $>150 \mathrm{mg} / \mathrm{dl}$ and low HDL-C when value $<40 \mathrm{mg} / \mathrm{dl}$. Dyslipidemia was defined by presence of one or more than one abnormal serum lipid concentration $^{[11]}$.

The atherogenic index of plasma (AIP) was calculated by the formula base 10 logarithm of the ratio of TG to HDL. An AIP of $>0.21$ was taken as high CAD risk, and $<0.21$ as low-intermediate CAD risk ${ }^{[12]}$.

Statistical analysis was carried out using Graph Pad Prism 6.0 version. Data were described as mean with S.D. for continuous variables and proportions for categorical variables. Fischer test was used to assess statistical significance of the difference between the percentages of poor glycemic control and lipid profile according to independent categorical variables. A $p$ value $<0.05$ was considered statistically significant.

\section{Results}

All the subjects were categorized into four groups. The first 3 groups show the effect of gender, age and duration of diabetes on HbA1c levels and lipid parameters, while the fourth group shows the influence of glycemic control on the circulating lipids. Out of a total of 278 diabetics, 130 were males and 148 were females. The mean age \pm SD of the male and female diabetic subjects were $54.71 \pm 11.40$ years and $56.34 \pm 10$ years, respectively. Although the levels of HbA1c, FPG were marginally higher in females and TG in male diabetics, they were not statistically significant. The total cholesterol and LDL cholesterol were significantly higher in females as compared to male diabetic. There was no significant difference in the HDL levels and the AIP between male and female diabetic subjects. [Table1].

[Table 2] shows the effect of age on HbA1c and lipid parameters. There appears a significant difference between TG levels and AIP ratio in relation to age. TG levels and AIP were raised in the subjects $<55$ years compared to those subjects >55 years. Although HbA1c and LDL levels are slightly raised in the lower age group these were not statistically significant. There was no difference in HDL levels among the two groups.

Comparison of the variables in relation to duration of diabetes $<6$ years vs $>6$ years[Table 3] shows that $\mathrm{HbA} 1 \mathrm{c}$, total cholesterol, TG, LDL cholesterol and AIP are elevated in the group with > 6 years duration of diabetes but only total cholesterol and LDL cholesterol were statistically significant. There is no difference in HDL levels among the two groups.

The mean \pm SD of the FPG and lipid parameters in relation to HbA1c are presented [Table 4]. All the lipid parameters except HDL cholesterol were statistically higher in the subjects with $\mathrm{HbA} 1 \mathrm{c}>7 \%$ as compared to the group with $\mathrm{HbA} 1 \mathrm{c}<7 \%$. These lipid variables were further categorized according to the NCEP (ATP III) criteria to better define the relationship of glycemic control with lipid parameters. Fischer exact paired test of proportion was used to calculate the p value. A highly statistically significant difference was observed [Table 5] for all the lipids, total cholesterol, TG, LDL levels and AIP except HDL cholesterol.

\section{Discussion}

Hyperlipidemia is common in diabetic patients. An association between hyperlipidemia and diabetic ketoacidosis was first demonstrated by Bloor in $1916^{[13]}$.

In our study, the distribution of characteristics according to gender showed that the total cholesterol and LDL cholesterol levels were significantly higher in females as compared to male diabetics. Although the levels of $\mathrm{HbA1c}$, were marginally higher in females than male diabetics, they were not statistically significant. 
Triglycerides were marginally elevated in the male diabetics but no difference was noted in the HDL-C and AIP.

The data of Table 1 shows that the mean age of diabetic females is higher as compared to males. Studies have shown that the disease shows a little gender preference, although diabetes becomes slightly more frequent in women with advancing age. The estrogen hormone, in females is said to have a protective role for developing diabetes ${ }^{[14]}$, Estrogen seems to contribute to glucose homeostasis in women by makes the body cells more receptive or sensitive to insulin ${ }^{[15]}$. Lipid, apoprotein, and lipoprotein profiles in women differ significantly from men and the effect of gender on lipid parameters is independent of age ${ }^{[16]}$.

While an elevation of total cholesterol and LDL-C in females and higher TG levels in males were in corroboration with other studies ${ }^{[17,22]}$, in contrast no difference was noted for HDL-C between the two genders.

Controversy exists regarding the effect of age, and duration of diabetes on lipid profile and glycemic status. While some studies reports no association, others have shown a significant correlation of these two factors with lipid profile ${ }^{[18,19]}$.

Our study shows that subjects less than 55years of age had statistically significant higher level of TG levels and AIP compared to the subjects above 55 years. HbA1c and LDL levels, even though elevated in the lower age group were are not statistically significant. HbA1c, total cholesterol, TG, LDL cholesterol and AIP seem to rise significantly with increased duration of diabetes. Longer duration of diabetes is known to be associated with poor control, possibly because of progressive impairment of insulin secretion with time because of beta cell failure, which makes the response to diet alone or oral agents unlikely ${ }^{[20]}$. Poor glycemic control, age-related pathology with duration of diabetes are thought to accelerate degenerative changes in a cooperative manner ${ }^{[21]}$.

The comparative effects of glycemic control in diabetic patients are shown in Table 5. Patients with worse glycemic control ( $\mathrm{HbA} 1 \mathrm{c}>7 \%)$ possessed significantly high total cholesterol and LDL as compared to patients having poor glycemic control. There was a linear and significant increase in triglycerides and AIP in the patients with impaired glycemic control. Our findings are in agreement with other studies ${ }^{[22,23] .}$ On the other hand, glycemic control had no impact on serum HDL levels. We report an increase in atherogenicity in the subjects with poor glycemic control.

Value of AIP corresponds closely to those of esterification rate in apo-B-lipoprotein - depleted plasma and to lipoprotein particle size. AIP reflects the delicate metabolic interactions within the whole lipoprotein complex $^{[12]}$.

\section{Conclusion}

The mean age (in years) of the subjects with poor glycemic control was lower, duration of diabetes longer and the levels of circulating lipids higher on comparison with subjects with good glycemic control.

Although hyperlipidemia and poor glycemic control are risk factors for cardiovascular disease as proven by other studies, there appears to be interplay among the various factors like age, duration, hyperlipidemia and hyperglycemia with eventual affect on the cardiac status. Onset of diabetes at an earlier age, longer duration of diabetes, poor glycemic control, development of insulin resistance produces an enhanced atherogenic environment within the circulation and probably account for the raised AIP in these cases. Infiltration of lipoproteins in arterial wall and dermal tissue has been implicated in atherosclerosis and xanthoma, respectively.

$\mathrm{HbAlc}$ and AIP appear to be a strong predictors for identifying atherogenicity and therefore a strong candidate for identifying subjects at risk for cardiovascular events.

\section{References}

[1] Whiting DR, Guariguata L, Weil C, Shaw J , IDF Diabetes Atlas: Global estimates of the prevalence of diabetes for 2011 and 2030 , Diabetes Res Clin Pract, 94, 2011, 311-321.

[2] Ronald K, Hyperglycemia and Microvascular and Macrovascular Disease in Diabetes, Diabetes Care, 18(2), 1995, 258-268.

[3] Irene M S, Amanda I A, Andrew W N, David R M, Susan EM et al, Association of glycaemia with macrovascular and microvascular complications of type 2 diabetes (UKPDS 35): prospective observational study, BMJ,321,2000,405-412.

[4] Kreisberg RA, Diabetic dyslipidemia, Am J Cardiol, 82(12A),1998, 67U-73U.

[5] Dobiásová M, AIP--atherogenic index of plasma as a significant predictor of cardiovascular risk: from research to practice. Vnitr Lek, 52(1), 2006, 64-71.

[6] Elizabeth S, Josef CSH, Golden,, Frederick L, Brancati,et al, Glycemic Control and Coronary Heart Disease Risk in Persons With and Without Diabetes. The Atherosclerosis Risk in Communities Study, Arch Intern Med, 165, 2005, 1910-1916

[7] Elizabeth S, Spyridon M, Gail B, Tejal R, Frederick L. Brancati, NR., Sherita HG, Meta-Analysis: Glycosylated Hemoglobin and Cardiovascular Disease in Diabetes Mellitus, Ann Intern Med 141(6), 2004, 421-431.

[8] Woodward A, Wallymahmed M, Wilding J, Gill G, Improved glycemic control: an unintended benefit of a nurse-led cardiovascular risk reduction clinic. Diabet Med, 22, 2005, 1272-1274.

[9] Nakamura K, Yamagishi SI, Adachi H, Kurita-Nakamura Y, Matsui T, Yoshida T, Sato A, Imaizumi T, Elevation of soluble form of receptor for advanced glycation end products (sRAGE) in diabetic subjects with coronary artery disease, Diabetes Metab Res $\operatorname{Rev} 23(5), 2007,368-371$.

[10] Position statement, Standards of Medical Care in Diabetes-2012, Diabetes Care, 35 (1), 2012, S11-S63. 
[11] Third Report of the Expert Panel on Detection, Evaluation, and Treatment of High Blood Cholesterol in Adults (Adult Treatment Panel III) http://www.nhlbi.nih.gov/guidelines/cholesterol/atp3full.pdf.

[12] Dobiasova_M, Frohlich J, The plasma parameter $\log ($ TG/HDL-C) as an atherogenic index: correlation with lipoprotein particle size and esterification rate in apoB-lipoprotein-depleted plasma (FER(HDL), Clin Biochem, 34(7), 2001, 583-588.

[13] Bloor W R., The lipoids of the blood in diabetes. J. Biol.Chem. 26, 1916, 417-30.

[14] Suhuan L, Franck MJ, Minireview: Estrogenic Protection of $\beta$-Cell Failure in Metabolic Diseases, Endocrinology, 151, 2010, $859-864$

[15] Mayumi, O., Seiji, N., Yoko, I., Eiko, Y. et al, . Effects of Post-menopausal Hormone Replacement Therapy on HbA1c levels, Diabetes care, 26, 2003, 1088-1092

[16] Tremollieres FA, Pouilles JM, Cauneille C, Ribot C, Coronary heart disease risk factors and menopause: a study in 1684 French women, Atherosclerosis 142(2), 1999, 415-23

[17] No authors listed] (1997) U.K. Prospective Diabetes Study 27, Plasma lipids and lipoproteins at diagnosis of NIDDM by age and sex. Diabetes Care 20, 1683-1687

[18] H.O. Otamere, C.P Aloamaka, PO.Okokhere, W.A. Adisa, Lipid Profile in Diabetes Mellitus; What Impact Has Age and Duration,. Br. J. Pharmacol. Toxicol, 2(3), 2011, 135-137,

[19] Amy SS, Lawrence M D, Thomas R K, Zhiqian G, Philip R K, Stephen R D, Elaine MU, Influence of Duration of Diabetes, Glycemic Control, and Traditional Cardiovascular Risk Factors on Early Atherosclerotic Vascular Changes in Adolescents and Young Adults with Type 2 Diabetes Mellitus. J Clin Endocrinol Metab 94, 2009, 3740-3745.

[20] Ronald K, Barbara EK, Scot E M, Relation of Glycemic Control to Diabetic Microvascular Complications in Diabetes Mellitus, Ann Intern Med, 124(1_Part_2), 1996, 90-96.

[21] David MN, The Pathophysiology of Diabetic Complications: How Much Does the Glucose Hypothesis Explain? Ann Intern Med, 124(1_Part_2), 1996, 86-89.

[22] Haseeb $\mathrm{AK}$, Clinical significance of $\mathrm{HbAlc}$ as a marker of circulating lipids in male and female type 2 diabetic patients Acta Diabetol,Springer-Verlag 2007.

[23] Ladeia AM, Adan L, Couto-Silva AC, Hiltner A, Guimaraes AC Lipid profile correlates with glycemic control in young patients with type 1 diabetes mellitus. Prev Cardiol, 9, 2006, 82-88.

TABLE 1: Characteristics of male and female diabetics

\begin{tabular}{|l|l|l|l|}
\hline variables & male & female & p value \\
\hline $\mathrm{N}$ & 130 & 148 & \\
\hline Age $(\mathrm{yrs})$ & $54.71 \pm 11.40$ & $56.34 \pm 10$ & 0.2051 \\
\hline Duration(yrs) & $5.17 \pm 3.80$ & $6.2 \pm 4.19$ & 0.0336 \\
\hline HbAlc $(\%)$ & $7.81 \pm 1.64$ & $8.06 \pm 1.77$ & 0.2251 \\
\hline FBS $(\mathrm{mg} / \mathrm{dl})$ & $161 \pm 60$ & $165 \pm 62$ & 0.5863 \\
\hline T cholesterol $(\mathrm{mgdl})$ & $182 \pm 47$ & $197 \pm 42$ & 0.0053 \\
\hline Triglycerides $(\mathrm{mg} / \mathrm{dl})$ & $198 \pm 133$ & $188 \pm 87$ & 0.4538 \\
\hline HDL cholesterol $(\mathrm{mg} / \mathrm{dl})$ & $38 \pm 1.89$ & $38 \pm 2.13$ & 1.000 \\
\hline LDL cholesterol $(\mathrm{mg} / \mathrm{dl})$ & $110 \pm 38$ & $123 \pm 32$ & 0.0022 \\
\hline AIP & $0.30 \pm 0.22$ & $0.30 \pm 0.18$ & 1.000 \\
\hline
\end{tabular}

statistically significant $-\mathrm{p}<0.05$

TABLE 2: Effect of age on HbA1c on lipid parameters

\begin{tabular}{|l|l|l|l|}
\hline Variables & $<55$ yrs & $>55 \mathrm{yrs}$ & pvalue \\
\hline $\mathrm{N}$ & 145 & 133 & \\
\hline Age & $47.45 \pm 6.74$ & $64.43 \pm 6.35$ & 0.001 \\
\hline Duration & $5.36 \pm 3.94$ & $6.1 \pm 4.12$ & 0.127 \\
\hline HbA1c $(\%)$ & $8.02 \pm 1.76$ & $7.9 \pm 1.66$ & 0.56 \\
\hline FBS(mg/dl) & $167 \pm 64$ & $160 \pm 57$ & 0.3381 \\
\hline T cholesterol(mg/dl) & $193 \pm 48$ & $187 \pm 41$ & 0.2655 \\
\hline Triglycerides $(\mathrm{mg} / \mathrm{dl})$ & $211 \pm 122$ & $173 \pm 93$ & 0.004 \\
\hline HDL cholesterol (mg/dl) & $38 \pm 2.01$ & $38 \pm 2.02$ & 1.000 \\
\hline LDL cholesterol (mg/dl) & $119 \pm 38$ & $115 \pm 34$ & 0.3574 \\
\hline AIP & $0.33 \pm 0.21$ & $0.26 \pm 0.19$ & 0.004 \\
\hline
\end{tabular}

statistically significant- $\mathrm{p}<0.05$ 
TABLE 3: Effect of duration of diabetes on HbA1c and lipid parameters

\begin{tabular}{|l|l|l|r|}
\hline & $<6 y r s$ & $>6 y r s$ & P value \\
\hline $\mathrm{N}$ & 184 & 94 & \\
\hline AGE & $54.6 \pm 10.32$ & $57.4 \pm 11.37$ & 0.0127 \\
\hline Duration & $3.3 \pm 1.80$ & $10.4 \pm 2.95$ & 0.0001 \\
\hline HbA1c & $7.88 \pm 1.73$ & $8.07 \pm 1.68$ & 0.2794 \\
\hline FPG & $162 \pm 68$ & $166 \pm 46$ & 0.5014 \\
\hline T chol & $187 \pm 41$ & $197 \pm 50$ & 0.0347 \\
\hline TG & $190 \pm 108$ & $199 \pm 115$ & 0.4339 \\
\hline HDL & $38 \pm 2.15$ & $38 \pm 1.75$ & 1 \\
\hline LDL & $114 \pm 32$ & $123 \pm 41$ & 0.0183 \\
\hline AIP & $0.29 \pm 0.20$ & $0.31 \pm 0.19$ & 0.3194 \\
\hline
\end{tabular}

statistically significant- $\mathrm{p}<0.05$

TABLE 4: Relation of HbA1c with lipid parameters in diabetics

\begin{tabular}{|l|l|l|l|}
\hline Parameter & $<7 \%$ & $>7 \%$ & pvalue \\
\hline $\mathrm{N}$ & 92 & 186 & \\
\hline Age & $56.8 \pm 10.95$ & $55 \pm 10.58$ & 0.1881 \\
\hline Duration & $4.49 \pm 3.46$ & $6.31 \pm 4.17$ & 0.0004 \\
\hline HbA1c $(\%)$ & $6.24 \pm 0.78$ & $8.79 \pm 1.39$ & 0.0001 \\
\hline FPG (mg/dl) & $117 \pm 22$ & $186 \pm 62$ & 0.0001 \\
\hline T cholesterol $(\mathrm{mg} / \mathrm{dl})$ & $169 \pm 30$ & $201 \pm 47$ & 0.0001 \\
\hline Triglycerides $(\mathrm{mg} / \mathrm{dl})$ & $155 \pm 62$ & $211 \pm 124$ & 0.0001 \\
\hline HDL cholesterol $(\mathrm{mg} / \mathrm{dl})$ & $38 \pm 1.84$ & $38 \pm 2.10$ & 1.000 \\
\hline LDL cholesterol $(\mathrm{mg} / \mathrm{dl})$ & $102 \pm 28$ & $124 \pm 37$ & 0.0001 \\
\hline AIP & $0.23 \pm 0.15$ & $0.33 \pm 0.22$ & 0.0001 \\
\hline
\end{tabular}

statistically significant- $\mathrm{p}<0.05$

TABLE 5: The comparative effects of glycemic control on lipid profile in diabetic patients

\begin{tabular}{|l|l|l|l|}
\hline Parameter & HbA1C $<7 \%$ & HbA1C $>7 \%$ & p value \\
\hline $\mathrm{N}$ & 92 & 186 & \\
\hline T. Cholesterol (mg/dl) & $74(80.4 \%)$ & $88(47.3)$ & 0.0001 \\
\hline$<200$ & $18(19.6)$ & $98(52.7)$ & \\
\hline$>200$ & 52 & 70 & 0.0032 \\
\hline Triglycerides (mg/dl) & 116 & \\
\hline$<150$ & 40 & 140 & 0.36 \\
\hline$>150$ & 74 & 46 & \\
\hline HDL Cholesterol (mg/dl) & 52 & 0.0001 \\
\hline$<40$ & 18 & 134 & \\
\hline$>40$ & 56 & 64 & 0.0136 \\
\hline LDL Cholesterol (mg/dl) & 122 \\
\hline$<100$ & 36 & 12 & \\
\hline$>100$ & 46 & 46 & \\
\hline AIP & 56 & \\
\hline$<0.21$ & \multicolumn{5}{|l|}{} \\
\hline$>0.21$ &
\end{tabular}

statistically significant- $\mathrm{p}<0.05$ 